\title{
Dynamical Model of Elementary Particles Based on an Analogy with Superconductivity. I*
}

\author{
Y. Nambu and G. Jona-Lasinio $\dagger$ \\ The Enrico Fermi Institute for Nuclear Studies and the Department of Physics, The University of Chicago, Chicago, Illinois
}

(Received October 27, 1960)

\begin{abstract}
It is suggested that the nucleon mass arises largely as a self-energy of some primary fermion field through the same mechanism as the appearance of energy gap in the theory of superconductivity. The idea can be put into a mathematical formulation utilizing a generalized Hartree-Fock approximation which regards real nucleons as quasi-particle excitations. We consider a simplified model of nonlinear four-fermion interaction which allows a $\gamma_{5}$-gauge group. An interesting consequence of the symmetry is that there arise automatically pseudoscalar zero-mass bound states of nucleon-antinucleon pair which may be regarded as an idealized pion. In addition, massive bound states of nucleon number zero and two are predicted in a simple approximation.

The theory contains two parameters which can be explicitly related to observed nucleon mass and the pion-nucleon coupling constant. Some paradoxical aspects of the theory in connection with the $\gamma_{5}$ transformation are discussed in detail.
\end{abstract}

\section{INTRODUCTION}

$I^{\mathrm{N}}$ $\mathrm{N}$ this paper we are going to develop a dynamical theory of elementary particles in which nucleons and mesons are derived in a unified way from a fundamental spinor field. ${ }^{1}$ In basic physical ideas, it has thus the characteristic features of a compound-particle model, but unlike most of the existing theories, dynamical treatment of the interaction makes up an essential part of the theory. Strange particles are not yet considered.

The scheme is motivated by the observation of an interesting analogy between the properties of Dirac particles and the quasi-particle excitations that appear in the theory of superconductivity, which was originated with great success by Bardeen, Cooper, and Schrieffer, ${ }^{2}$ and subsequently given an elegant mathematical formulation by Bogoliubov. ${ }^{3}$ The characteristic feature of the BCS theory is that it produces an energy gap between the ground state and the excited states of a superconductor, a fact which has been confirmed experimentally. The gap is caused due to the fact that the attractive phonon-mediated interaction between electrons produces correlated pairs of electrons with opposite momenta and spin near the Fermi surface, and it takes a finite amount of energy to break this correlation.

Elementary excitations in a superconductor can be conveniently described by means of a coherent mixture of electrons and holes, which obeys the following

\footnotetext{
* Supported by the U. S. Atomic Energy Commission.

† Fulbright Fellow, on leave of absence from Instituto di Fisica dell' Universita, Roma, Italy and Istituto Nazionale di Fisica Nucleare, Sezione di Roma, Italy.

1 A preliminary version of the work was presented at the Midwestern Conference on Theoretical Physics, April, 1960 (unpublished). See also Y. Nambu, Phys. Rev. Letters 4, 380 (1960); and Proceedings of the Tenth Annual Rochester Conference on High-Energy Nuclear Physics, 1960 (to be published).

${ }_{2}^{2}$ J. Bardeen, L. N. Cooper, and J. R. Schrieffer, Phys. Rev. 106, 162 (1957).

${ }^{3}$ N. N. Bogoliubov, J. Exptl. Theoret. Phys. (U.S.S.R.) 34, 58, 73 (1958) [translation: Soviet Phys.-JETP 34, 41, 51 (1958)]; N. N. Bogoliubov, V. V. Tolmachev, and D. V. Shirkov, A New Method in the Theory of Superconductivity (Academy of Sciences of U.S.S.R., Moscow, 1958).
}

equations $s^{3,4}$ :

$$
\begin{gathered}
E \psi_{p+}=\epsilon_{p} \psi_{p+}+\phi \psi_{-p-} * \\
E \psi_{-p-} *=-\epsilon_{p} \psi_{-p-} *+\phi \psi_{p+},
\end{gathered}
$$

near the Fermi surface. $\psi_{p+}$ is the component of the excitation corresponding to an electron state of momentum $p$ and spin + (up), and $\psi_{-p-}{ }^{*}$ corresponding to a hole state of momentum $p$ and spin + , which means an absence of an electron of momentum $-p$ and spin - (down). $\epsilon_{p}$ is the kinetic energy measured from the Fermi surface; $\phi$ is a constant. There will also be an equation complex conjugate to Eq. (1), describing another type of excitation.

Equation (1) gives the eigenvalues

$$
E_{p}= \pm\left(\epsilon_{p}^{2}+\phi^{2}\right)^{\frac{1}{2}} \text {. }
$$

The two states of this quasi-particle are separated in energy by $2\left|E_{p}\right|$. In the ground state of the system all the quasi-particles should be in the lower (negative) energy states of Eq. (2), and it would take a finite energy $2\left|E_{p}\right| \geqslant 2|\phi|$ to excite a particle to the upper state. The situation bears a remarkable resemblance to the case of a Dirac particle. The four-component Dirac equation can be split into two sets to read

$$
\begin{aligned}
E \psi_{1} & =\sigma \cdot p \psi_{1}+m \psi_{2}, \\
E \psi_{2} & =-\sigma \cdot p \psi_{2}+m \psi_{1}, \\
E_{p} & = \pm\left(p^{2}+m^{2}\right)^{\frac{1}{2}},
\end{aligned}
$$

where $\psi_{1}$ and $\psi_{2}$ are the two eigenstates of the chirality operator $\gamma_{5}=\gamma_{1} \gamma_{2} \gamma_{3} \gamma_{4}$.

According to Dirac's original interpretation, the ground state (vacuum) of the world has all the electrons in the negative energy states, and to create excited states (with zero particle number) we have to supply an energy $\geqslant 2 m$.

In the BCS-Bogoliubov theory, the gap parameter $\phi$, which is absent for free electrons, is determined essentially as a self-consistent (Hartree-Fock) representation of the electron-electron interaction effect.

\footnotetext{
${ }^{4}$ J. G. Valatin, Nuovo cimento 7, 843 (1958).
} 
One finds that

$$
\phi \approx \omega \exp [-1 / \rho]
$$

where $\omega$ is the energy bandwidth ( $\approx$ the Debye frequency) around the Fermi surface within which the interaction is important; $\rho$ is the average interaction energy of an electron interacting with unit energy shell of electrons on the Fermi surface. It is significant that $\phi$ depends on the strength of the interaction (coupling constant) in a nonanalytic way.

We would like to pursue this analogy mathematically. As the energy gap $\phi$ in a superconductor is created by the interaction, let us assume that the mass of a Dirac particle is also due to some interaction between massless bare fermions. A quasi-particle in a superconductor is a mixture of bare electrons with opposite electric charges (a particle and a hole) but with the same spin; correspondingly a massive Dirac particle is a mixture of bare fermions with opposite chiralities, but with the same charge or fermion number. Without the gap $\phi$ or the mass $m$, the respective particle would become an eigenstate of electric charge or chirality.

Once we make this analogy, we immediately notice further consequences of special interest. It has been pointed out by several people ${ }^{3,5-8}$ that in a refined theory of superconductivity there emerge, in addition to the individual quasi-particle excitations, collective excitations of quasi-particle pairs. (These can alternatively be interpreted as moving states of bare electron pairs which are originally precipitated into the ground state of the system.) In the absence of Coulomb interaction, these excitations are phonon-like, filling the gap of the quasi-particle spectrum.

In general, they are excited when a quasi-particle is accelerated in the medium, and play the role of a backflow around the particle, compensating the change of charge localized on the quasi-particle wave packet. Thus these excitations are necessary consequences of the fact that individual quasi-particles are not eigenstates of electric charge, and hence their equations are not gauge invariant; whereas a complete description of the system must be gauge invariant. The logical connection between gauge invariance and the existence of collective states has been particularly emphasized by one of the authors. ${ }^{8}$

This observation leads to the conclusion that if a Dirac particle is actually a quasi-particle, which is only an approximate description of an entire system where chirality is conserved, then there must also exist collective excitations of bound quasi-particle pairs. The chirality conservation implies the invariance of the theory under the so-called $\gamma_{5}$ gauge group, and from its nature one can show that the collective state must be a pseudoscalar quantity.

\footnotetext{
${ }^{5}$ D. Pines and J. R. Schrieffer, Nuovo cimento 10, 496 (1958)

${ }^{6}$ P. W. Anderson, Phys. Rev. 110, 827, 1900 (1958); 114, 1002 (1959).

${ }^{7}$ G. Rickayzen, Phys. Rev. 115, 795 (1959).

${ }^{8}$ Y. Nambu, Phys. Rev. 117, 648 (1960).
}

It is perhaps not a coincidence that there exists such an entity in the form of the pion. For this reason, we would like to regard our theory as dealing with nucleons and mesons. The implication would be that the nucleon mass is a manifestation of some unknown primary interaction between originally massless fermions, the same interaction also being responsible for the binding of nucleon pairs into pions.

An additional support of the idea can be found in the weak decay processes of nucleons and pions which indicate that the $\gamma_{5}$ invariance is at least approximately conserved, as will be discussed in Part II. There are some difficulties, however, that naturally arise on further examination.

Comparison between a relativistic theory and a nonrelativistic, intuitive picture is often dangerous, because the former is severely restricted by the requirement of relativistic invariance. In our case, the energy-gap equation (4) depends on the energy density on the Fermi surface; for zero Fermi radius, the gap vanishes. The Fermi sphere, however, is not a relativistically invariant object, so that in the theory of nucleons it is not clear whether a formula like Eq. (4) could be obtained for the mass. This is not surprising, since there is a well known counterpart in classical electron theory that a finite electron radius is incompatible with relativistic invariance.

We avoid this difficulty by simply introducing a relativistic cutoff which takes the place of the Fermi sphere. Our framework does not yet resolve the divergence difficulty of self-energy, and the origin of such an effective cutoff has to be left as an open question.

The second difficulty concerns the mass of the pion. If pion is to be identified with the phonon-like excitations associated with a gauge group, its mass must necessarily be zero. It is true that in real superconductors the collective charge fluctuation is screened by Coulomb interaction to turn into the plasma mode, which has a finite "rest mass." A similar mechanism may be operating in the meson case too. It is possible, however, that the finite meson mass means that chirality conservation is only approximate in a real theory. From the evidence in weak interactions, we are inclined toward the second view.

The observation made so far does not yet give us a clue as to the exact mechanism of the primary interaction. Neither do we have a fundamental understanding of the isospin and strangeness quantum numbers, although it is easy to incorporate at least the isospin degree of freedom into the theory from the beginning. The best we can do here is to examine the various existing models for their logical simplicity and experimental support, if any. We will do this in Sec. 2, and settle for the moment on a nonlinear four-fermion interaction of the Heisenberg type. For reasons of simplicity in presentation, we adopt a model without isospin and strangeness degrees of freedom, and possessing complete $\gamma_{5}$ invariance. Once the choice is made, 
we can explore the whole idea mathematically, using essentially the formulation developed in reference 8. It is gratifying that the various field-theoretical techniques can be fully utilized. Section 3 will be devoted to introduction of the Hartree-Fock equation for nucleon selfenergy, which will make the starting point of the theory. Then we go on to discuss in Sec. 4 the collective modes. In addition to the expected pseudoscalar "pion" states, we find other massive mesons of scalar and vector variety, as well as a scalar "deuteron." The coupling constants of these mesons can be easily determined. The relation of the pion to the $\gamma_{5}$ gauge group will be discussed in Secs. 5 and 6.

The theory promises many practical consequences. For this purpose, however, it is necessary to make our model more realistic by incorporating the isospin, and allowing for a violation of $\gamma_{5}$ invariance. But in doing so, there arise at the same time new problems concerning the mass splitting and instability. This refined model will be elaborated in Part II of this work, where we shall also find predictions about strong and weak interactions. Thus the general structure of the weak interaction currents modified by strong interactions can be treated to some degree, enabling one to derive the decay processes of various particles under simple assumptions. The calculation of the pion decay rate gives perhaps one of the most interesting supports of the theory. Results about strong interactions themselves are equally interesting. We shall find specific predictions about heavier mesons, which are in line with the recent theoretical expectations.

\section{THE PRIMARY INTERACTION}

We briefly discuss the possible nature of the primary interaction between fermions. Lacking any radically new concepts, the interaction could be either mediated by some fundamental Bose field or due to an inherent nonlinearity in the fermion field. According to our postulate, these interactions must allow chirality conservation in addition to the conservation of nucleon number. The chirality $X$ here is defined as the eigenvalue of $\gamma_{5}$, or in terms of quantized fields,

$$
X=\int \bar{\psi} \gamma_{4} \gamma_{5} \psi d^{3} x
$$

The nucleon number is, on the other hand

$$
N=\int \bar{\psi} \gamma_{4} \psi d^{3} x
$$

These are, respectively, generators of the $\gamma_{5^{-}}$and ordinary-gauge groups

$$
\begin{aligned}
& \psi \rightarrow \exp \left[i \alpha \gamma_{5}\right] \psi, \quad \bar{\psi} \rightarrow \bar{\psi} \exp \left[i \alpha \gamma_{5}\right] \\
& \psi \rightarrow \exp [i \alpha] \psi, \quad \bar{\psi} \rightarrow \bar{\psi} \exp [-i \alpha]
\end{aligned}
$$

where $\alpha$ is an arbitrary constant phase.
Furthermore, the dynamics of our theory would require that the interaction be attractive between particle and antiparticle in order to make bound-state formation possible. Under the transformation (2.3), various tensors transform as follows:

$$
\begin{array}{ll}
\text { Vector: } & i \bar{\psi} \gamma_{\mu} \psi \rightarrow i \bar{\psi} \gamma_{\mu} \psi, \\
\text { Axial vector: } & i \bar{\psi} \gamma_{\mu} \gamma_{5} \psi \rightarrow i \bar{\psi} \gamma_{\mu} \gamma_{5} \psi, \\
\text { Scalar: } & \bar{\psi} \psi \rightarrow \bar{\psi} \psi \cos 2 \alpha+i \bar{\psi} \gamma_{5} \psi \sin 2 \alpha \\
\text { Pseudoscalar: }: & i \bar{\psi} \gamma_{5} \psi \rightarrow i \bar{\psi} \gamma_{5} \psi \cos 2 \alpha-\bar{\psi} \psi \sin 2 \alpha \\
\text { Tensor: } & \bar{\psi} \sigma_{\mu \nu} \psi \rightarrow \bar{\psi} \sigma_{\mu \nu} \psi \cos 2 \alpha+i \bar{\psi} \gamma_{5} \sigma_{\mu \nu} \psi \sin 2 \alpha
\end{array}
$$

It is obvious that a vector or pseudovector Bose field coupled to the fermion field satisfies the invariance. The vector case would also satisfy the dynamical requirement since, as in the electromagnetic interaction, the forces would be attractive between opposite nucleon charges. The pseudovector field, on the other hand, does not meet the requirement as can be seen by studying the self-consistent mass equation discussed later.

The vector field looks particularly attractive since it can be associated with the nucleon number gauge group. This idea has been explored by Lee and Yang, ${ }^{9}$ and recently by Sakurai. ${ }^{10}$ But since we are dealing with strong interactions, such a field would have to have a finite observed mass in a realistic theory. Whether this is compatible with the invariance requirement is not yet clear. (Besides, if the bare mass of both spinor and vector field were zero, the theory would not contain any parameter with the dimensions of mass.)

The nonlinear fermion interaction seems to offer another possibility. Heisenberg and his co-workers ${ }^{11}$ have been developing a comprehensive theory of elementary particles along this line. It is not easy, however, to gain a clear physical insight into their results obtained by means of highly complicated mathematical machinery.

We would like to choose the nonlinear interaction in this paper. Although this looks similar to Heisenberg's theory, the dynamical treatment will be quite different and more amenable to qualitative understanding.

The following Lagrangian density will be assumed $(\hbar=c=1)$ :

$$
L=-\bar{\psi} \gamma_{\mu} \partial_{\mu} \psi+g_{0}\left[(\bar{\psi} \psi)^{2}-\left(\bar{\psi} \gamma_{5} \psi\right)^{2}\right]
$$

The coupling parameter $g_{0}$ is positive, and has dimensions $[\text { mass }]^{-2}$. The $\gamma_{5}$ invariance property of the interaction is evident from Eq. (2.5). According to the Fierz theorem, it is also equivalent to

$$
-\frac{1}{2} g_{0}\left[\left(\bar{\psi} \gamma_{\mu} \psi\right)^{2}-\left(\bar{\psi} \gamma_{\mu} \gamma_{5} \psi\right)^{2}\right]
$$

This particular choice of $\gamma_{5}$-invariant form was taken without a compelling reason, but has the advantage

\footnotetext{
${ }^{9}$ T. D. Lee and C. N. Yang, Phys. Rev. 98, 1501 (1955).

${ }^{10} \mathrm{~J}$. J. Sakurai, Ann. Phys. 11, 1 (1960).

${ }^{11}$ W. Heisenberg, Z. Naturforsch. 14, 441 (1959). Earlier papers are quoted there.
} 
that it can be naturally extended to incorporate isotopic spin. ${ }^{12}$

Unlike Heisenberg's case, we do not have any theory about the handling of the highly divergent singularities inherent in nonlinear interactions. So we will introduce, as an additional and independent assumption, an ad hoc relativistic cutoff or form factor in actual calculations. Thus the theory may also be regarded as an approximate treatment of the intermediate-boson model with a large effective mass.

As will be seen in subsequent sections, the nonlinear model makes mathematics particularly easy, at least in the lowest approximation, enabling one to derive many interesting quantitative results.

\section{THE SELF-CONSISTENT EQUATION FOR NUCLEON MASS}

We will assume that all quantities we calculate here are somewhow convergent, without asking the reason behind it. This will be done actually by introducing a suitable phenomenological cutoff.

Without specifying the interaction, let $\Sigma$ be the unrenormalized proper self-energy part of the fermion, expressed in terms of observed mass $m$, coupling constant $g$, and cutoff $\Lambda$. A real Dirac particle will satisfy the equation

$$
i \gamma \cdot p+m_{0}+\Sigma(p, m, g, \Lambda)=0
$$

for $i \gamma \cdot p+m=0$. Namely

$$
m-m_{0}=\left.\Sigma(p, m, g, \Lambda)\right|_{i \gamma \cdot p+m=0} .
$$

The $g$ will also be related to the bare coupling $g_{0}$ by an equation of the type

$$
g / g_{0}=\Gamma(m, g, \Lambda) .
$$

Equations (3.1) and (3.2) may be solved by successive approximation starting from $m_{0}$ and $g_{0}$. It is possible, however, that there are also solutions which cannot thus be obtained. In fact, there can be a solution $m \neq 0$ even in the case where $m_{0}=0$, and moreover the symmetry seems to forbid a finite $m$.

This kind of situation can be most easily examined by means of the generalized Hartree-Fock procedure ${ }^{8,13}$ which was developed before in connection with the theory of superconductivity. The basic idea is not new in field theory, and in fact in its simplest form the method is identical with the renormalization procedure of Dyson, considered only in a somewhat different context.

Suppose a Lagrangian is composed of the free and interaction part: $L=L_{0}+L_{i}$. Instead of diagonalizing $L_{0}$ and treating $L_{i}$ as perturbation, we introduce the self-

\footnotetext{
12 This will be done in Part II.

${ }^{13}$ N. N. Bogoliubov, Uspekhi Fiz. Nauk 67, 549 (1959) [translation: Soviet Phys.-Uspekhi 67, 236 (1959)].
}

energy Lagrangian $L_{s}$, and split $L$ thus

$$
\begin{aligned}
L & =\left(L_{0}+L_{s}\right)+\left(L_{i}-L_{s}\right) \\
& =L_{0}{ }^{\prime}+L_{i}{ }^{\prime} .
\end{aligned}
$$

For $L_{s}$ we assume quite general form (quadratic or bilinear in the fields) such that $L_{0}^{\prime}$ leads to linear field equations. This will enable one to define a vacuum and a complete set of "quasi-particle" states, each particle being an eigenmode of $L_{0}{ }^{\prime}$. Now we treat $L_{i}^{\prime}$ as perturbation, and determine $L_{s}$ from the requirement that $L_{i}^{\prime}$ shall not yield additional self-energy effects. This procedure then leads to Eq. (3.2). The self-consistent nature of such a procedure is evident since the selfenergy is calculated by perturbation theory with fields which are already subject to the self-energy effect.

In order to apply the method to our problem, let us assume that $L_{s}=-m \bar{\psi} \psi$, and introduce the propagator $S_{F}{ }^{(m)}(x)$ for the corresponding Dirac particle with mass $m$. In the lowest order, and using the two alternative forms Eqs. (2.6) and (2.7), we get for Eq. (3.2)

$$
\begin{aligned}
\Sigma= & 2 g_{0}\left[\operatorname{Tr} S_{F}^{(m)}(0)-\gamma_{5} \operatorname{Tr} S_{F}^{(m)}(0) \gamma_{5}\right. \\
& \left.-\frac{1}{2} \gamma_{\mu} \operatorname{Tr} \gamma_{\mu} S_{F}^{(m)}(0)+\frac{1}{2} \gamma_{\mu} \gamma_{5} \operatorname{Tr} \gamma_{\mu} \gamma_{5} S_{F}^{(m)}(0)\right]
\end{aligned}
$$

in coordinate space.

This is quadratically divergent, but with a cutoff can be made finite. In momentum space we have

$$
\Sigma=-\frac{8 g_{0} i}{(2 \pi)^{4}} \int \frac{m}{p^{2}+m^{2}-i \epsilon} d^{4} p F(p, \Lambda),
$$

where $F(p, \Lambda)$ is a cutoff factor. In this case the selfenergy operator is a constant. Substituting $\Sigma$ from Eq. (3.5), Eq. (3.2) gives $\left(m_{0}=0\right)$

$$
m=-\frac{g_{0} m i}{2 \pi^{4}} \int \frac{d^{4} p}{p^{2}+m^{2}-i \epsilon} F(p, \Lambda) .
$$

This has two solutions: either $m=0$, or

$$
1=-\frac{g_{0} i}{2 \pi^{4}} \int \frac{d^{4} p}{p^{2}+m^{2}-i \epsilon} F(p, \Lambda) .
$$

The first trivial one corresponds to the ordinary perturbative result. The second, nontrivial solution will determine $m$ in terms of $g_{0}$ and $\Lambda$.

If we evaluate Eq. (3.7) with a straight noninvariant cutoff at $|\mathbf{p}|=\Lambda$, we get

$$
\frac{\pi^{2}}{g_{0} \Lambda^{2}}=\left(\frac{m^{2}}{\Lambda^{2}}+1\right)^{\frac{1}{2}}-\frac{m^{2}}{\Lambda^{2}} \ln \left[\left(\frac{\Lambda^{2}}{m^{2}}+1\right)^{\frac{1}{2}}+\frac{\Lambda}{m}\right] .
$$

If we use Eq. (3.5) with an invariant cutoff at $p^{2}=\Lambda^{2}$ after the change of path: $p_{0} \rightarrow i p_{0}$, we get

$$
\frac{2 \pi^{2}}{g_{0} \Lambda^{2}}=1-\frac{m^{2}}{\Lambda^{2}} \ln \left(\frac{\Lambda^{2}}{m^{2}}+1\right) .
$$


Since the right-hand side of Eq. (3.8) or (3.9) is positive and $\leqslant 1$ for real $\Lambda / m$, the nontrivial solution exists only if

$$
0<2 \pi^{2} / g_{0} \Lambda^{2}<1 \text {. }
$$

Equation (3.9) is plotted in Fig. 1 as a function of $m^{2} / \Lambda^{2}$. As $g_{0} \Lambda^{2}$ increases over the critical value $2 \pi^{2}, m$ starts rising from 0 . The nonanalytic nature of the solution is evident as $m$ cannot be expanded in powers of $g_{0}$.

In the following we will assume that Eq. (3.10) is satisfied, so that the nontrivial solution exists. As we shall see later, physically this means that the nucleonantinucleon interaction must be attractive $\left(g_{0}>0\right)$ and strong enough to cause a bound pair of zero total mass. In the BCS theory, the nontrivial solution corresponds to a superconductive state, whereas the trivial one corresponds to a normal state, which is not the true ground state of the superconductor. We may expect a similar situation to hold in the present case.

In this connection, it must be kept in mind that our solutions are only approximate ones. We are operating under the assumption that the corrections to them are not catastrophic, and can be appropriately calculated when necessary. If this does not turn out to be so for some solution, such a solution must be discarded. Later we shall indeed find this possibility for the trivial solution, but for the moment we will ignore such considerations.

Let us define then the vacuum corresponding to the two solutions. Let $\psi^{(0)}$ and $\psi^{(m)}$ be quantized fields satisfying the equations

$$
\begin{gathered}
\gamma_{\mu} \partial_{\mu} \psi^{(0)}(x)=0, \\
\left(\gamma_{\mu} \partial_{\mu}+m\right) \psi^{(m)}(x)=0 \\
\psi^{(0)}(x)=\psi^{(m)}(x) \text { for } x_{0}=0 .
\end{gathered}
$$

According to the standard procedure, we decompose the $\psi$ 's into Fourier components :

$$
\begin{aligned}
& \psi_{\alpha}^{(i)}(x)=\frac{1}{V^{\frac{1}{2}}} \sum_{\substack{\mathbf{p}, s \\
p_{0}=\left(p^{2}+m^{2}\right)^{\frac{3}{2}}}}\left[u_{\alpha}^{(i)}(\mathbf{p}, s) a^{(i)}(\mathbf{p}, s) e^{i p \cdot x}\right. \\
& \left.+v_{\alpha}^{*(i)}(\mathbf{p}, s) b^{(i) \dagger}(\mathbf{p}, s) e^{-i p \cdot x}\right],
\end{aligned}
$$

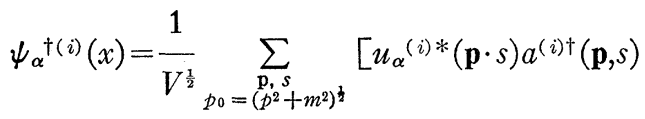

$$
\begin{aligned}
& \left.\times e^{-i p \cdot x}+v_{\alpha}^{(i)}(\mathbf{p}, s) b^{(i)}(\mathbf{p}, s) e^{i p \cdot x}\right], \\
& i=0 \text { or } m \text {, }
\end{aligned}
$$

where $u_{\alpha}{ }^{(i)}(\mathbf{p}, s), v_{\alpha}{ }^{(i)}(\mathbf{p}, s)$ are the normalized spinor eigenfunctions for particles and antiparticles, with momentum $p$ and helicity $s= \pm 1$, and

$$
\begin{aligned}
\left\{a^{(i)}(\mathbf{p}, s), a^{(i) \dagger}\left(\mathbf{p}^{\prime}, s^{\prime}\right)\right\} \\
=\left\{b^{(i)}(\mathbf{p}, s), b^{(i) \dagger}\left(\mathbf{p}^{\prime}, s^{\prime}\right)\right\}=\delta_{\mathrm{pp}^{\prime}} \delta_{s s^{\prime}}, \text { etc. }
\end{aligned}
$$

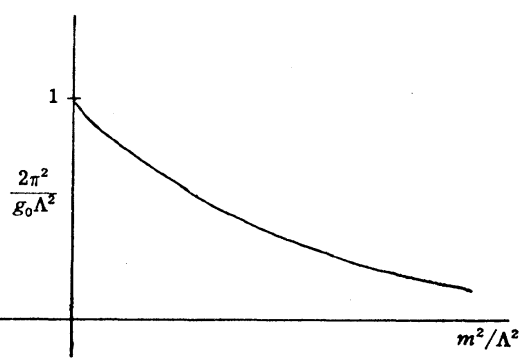

FIG. 1. Plot of the self-consistent mass equation (3.9).

The operator sets $\left(a^{(0)}, b^{(0)}\right)$ and $\left(a^{(m)}, b^{(m)}\right)$ are related by a canonical transformation because of Eq. (3.11c):

$$
\begin{aligned}
a^{(m)}(\mathbf{p}, s)= & \sum_{\alpha, s^{\prime}}\left[u_{\alpha}{ }^{(m) *}(\mathbf{p}, s) u_{\alpha}{ }^{(0)}\left(\mathbf{p}, s^{\prime}\right) a^{(0)}\left(\mathbf{p}, s^{\prime}\right)\right. \\
& \left.+u_{\alpha}{ }^{(m) *}(\mathbf{p}, s) v_{\alpha}{ }^{(0) *}\left(-\mathbf{p}, s^{\prime}\right) b^{(0) \dagger}\left(-\mathbf{p}, s^{\prime}\right)\right] \\
b^{(m)}(\mathbf{p}, s)= & \sum_{\alpha, s^{\prime}}\left[v_{\alpha}{ }^{(m) *}(\mathbf{p}, s) v_{\alpha}^{(0)}\left(\mathbf{p}, s^{\prime}\right) b^{(0)}\left(\mathbf{p}, s^{\prime}\right)\right. \\
& \left.+v_{\alpha}{ }^{(m) *}(\mathbf{p}, s) u_{\alpha}{ }^{(0) *}\left(-\mathbf{p}, s^{\prime}\right) a^{(0) \dagger}\left(-\mathbf{p}, s^{\prime}\right)\right] .
\end{aligned}
$$

Using Eq. (1.3), this is evaluated to give

$$
\begin{aligned}
& a^{(m)}(\mathbf{p}, s)= {\left[\frac{1}{2}\left(1+\beta_{p}\right)\right]^{\frac{1}{2}} a^{(0)}(\mathbf{p}, s) } \\
&++\left[\frac{1}{2}\left(1-\beta_{p}\right)\right]^{\frac{1}{2}} b^{(0) \dagger}(-\mathbf{p}, s), \\
& b^{(m)}(\mathbf{p}, s)= {\left[\frac{1}{2}\left(1+\beta_{p}\right)\right]^{\frac{1}{2}} b^{(0)}(\mathbf{p}, s) } \\
&--\left[\frac{1}{2}\left(1-\beta_{p}\right)\right]^{\frac{1}{2}} a^{(0) \dagger}(-\mathbf{p}, s), \\
& \beta_{p}=|\mathbf{p}| /\left(\mathbf{p}^{2}+m^{2}\right)^{\frac{1}{2}}
\end{aligned}
$$

The vacuum $\Omega^{(0)}$ or $\Omega^{(m)}$ with respect to the field $\psi^{(0)}$ or $\psi^{(m)}$ is now defined as

$$
\begin{gathered}
a^{(0)}(\mathbf{p}, s) \Omega^{(0)}=b^{(0)}(\mathbf{p} \xi s) \Omega^{(0)}=0, \\
a^{(m)}(\mathbf{p}, s) \Omega^{(m)}=b^{(m)}(\mathbf{p}, s) \Omega^{(m)}=0 .
\end{gathered}
$$

Both $\psi^{(0)}, \psi^{(0)}$ and $\psi^{(m)}, \psi^{(m)}$ applied to $\Omega^{(0)}$ always create particles of mass zero, whereas the same applied to $\Omega^{(m)}$ create particles of mass $m$.

From Eqs. (3.15) and (3.16) we obtain

$$
\begin{aligned}
\Omega^{(m)}=\prod_{\mathbf{p}, s}\left\{\left[\frac{1}{2}\left(1+\beta_{p}\right)\right]^{\frac{1}{2}}\right. \\
\left.-\left[\frac{1}{2}\left(1-\beta_{p}\right)\right]^{\frac{1}{2}} a^{(0) \dagger}(\mathbf{p}, s) b^{(0) \dagger}(-\mathbf{p}, s)\right\} \Omega^{(0)} .
\end{aligned}
$$

Thus $\Omega^{(m)}$ is, in terms of zero-mass particles, a superposition of pair states. Each pair has zero momentum, spin and nucleon number, and carries \pm 2 units of chirality, since chirality equals minus the helicity $s$ for massless particles.

Let us calculate the scalar product $\left(\Omega^{(0)}, \Omega^{(m)}\right)$ from Eq. (3.15) :

$$
\begin{aligned}
\left(\Omega^{(0)}, \Omega^{(m)}\right) & =\prod_{\mathbf{p}, s}\left[\frac{1}{2}\left(1+\beta_{p}\right)\right]^{\frac{1}{2}} \\
& =\exp \left\{\sum_{\mathbf{p}, s} \frac{1}{2} \ln \left[\frac{1}{2}\left(1+\beta_{p}\right)\right]\right\} .
\end{aligned}
$$

For large $p, \beta_{\boldsymbol{p}} \sim 1-m^{2} / 2 \mathbf{p}^{2}$, so that the exponent 
diverges as $V \pi m^{2} \int d \mathbf{p} /(2 \pi)^{3}(V=$ normalization volume). Hence

$$
\left(\Omega^{(0)}, \Omega^{(m)}\right)=0 .
$$

It is easy to see that any two states $\Psi^{(0)}$ and $\Psi^{(m)}$, obtained by applying a finite number of creation operators on $\Omega^{(0)}$ and $\Omega^{(m)}$ respectively, are also orthogonal.

Thus the two "worlds" based on $\Omega^{(0)}$ and $\Omega^{(m)}$ are physically distinct and outside of each other. No interaction or measurement, in the usual sense, can bridge them in finite steps.

What is the energy difference of the two vacua? Since both are Lorentz invariant states, the difference can only be either zero or infinity. Using the expression

$$
\begin{aligned}
H^{(m)}=\sum_{\mathbf{p}, s}\left(\mathbf{p}^{2}+m^{2}\right)^{\frac{1}{2}}\left\{a^{(m) \dagger}(\mathbf{p}, s) a^{(m)}(\mathbf{p}, s)\right. & \\
& \left.-b^{(m)}(\mathbf{p}, s) b^{(m) \dagger}(\mathbf{p}, s)\right\}, \\
H^{(0)}=\sum_{\mathbf{p}, s}|\mathbf{p}|\left\{a^{(0) \dagger}(\mathbf{p}, s) a^{(0)}(\mathbf{p}, s)\right. & \\
& \left.\quad-b^{(0)}(\mathbf{p}, s) b^{(0) \dagger}(\mathbf{p}, s)\right\},
\end{aligned}
$$

we get for the respective energies

$$
E^{(m)}-E^{(0)}=-2 \sum_{\mathbf{p}}\left[\left(\mathbf{p}^{2}+m^{2}\right)^{\frac{1}{2}}-|\mathbf{p}|\right],
$$

which is negative and quadratically divergent. So $\Omega^{(m)}$ may be called the "true" ground state, as was expected.

There remains finally the question of $\gamma_{5}$ invariance. The original Hamiltonian allowed two conservations $X$ and $N$, Eqs. (2.1) and (2.2). Both $\Omega^{(0)}$ and $\Omega^{(m)}$ belong to $N=0$, and their elementary excitations carry $N= \pm 1$. In the case of $X$, the same is true for the space $\Omega^{(0)}$, but $\Omega^{(m)}$ as well as its elementary excitations are not eigenstates of $X$, as is clear from the foregoing results. If the latter solution is to be a possibility, there must be an infinite degeneracy* with respect to the quantum number $X$. A ground state will be in general a linear combination of degenerate states with different $X=0$, $\pm 2, \cdots$ :

$$
\Omega^{(m)}=\sum_{n=-\infty}^{\infty} C_{2 n} \Omega_{2 n}{ }^{(m)} .
$$

Equation (3.17) is in fact a particular case of this. The $\gamma_{5}$-gauge transformation Eq. (2.3) induces the change

$$
\begin{gathered}
a^{(0)}(\mathbf{p}, \pm 1) \rightarrow e^{\mp i \alpha} a^{(0)}(\mathbf{p}, \pm 1) \\
b^{(0)}(\mathbf{p}, \pm 1) \rightarrow e^{\mp i \alpha} b^{(0)}(\mathbf{p}, \pm 1) \\
a^{(0) \dagger}(\mathbf{p}, \pm 1) \rightarrow e^{ \pm i \alpha} a^{(0) \dagger}(\mathbf{p}, \pm 1) \\
b^{(0) \dagger}(\mathbf{p}, \pm 1) \rightarrow e^{ \pm i \alpha} b^{(0) \dagger}(\mathbf{p}, \pm 1)
\end{gathered}
$$

and the coefficients of Eq. (3.22) become

In particular

$$
C_{2 n} \rightarrow e^{-2 n i \alpha} C_{2 n}
$$

$$
\begin{aligned}
\Omega^{(m)} \rightarrow & \Omega_{\alpha}^{(m)} \\
= & \exp [-i \alpha X] \Omega^{(m)} \\
= & \prod_{\mathbf{p}, \pm}\left\{\left[\frac{1}{2}\left(1+\beta_{p}\right)\right]^{\frac{1}{2}}-\left[\frac{1}{2}\left(1-\beta_{p}\right)\right]^{\frac{1}{2}}\right. \\
& \left.\quad \times e^{ \pm 2 i \alpha} a^{(0) \dagger}(p, \pm) b^{(0) \dagger}(-p, \pm)\right\} \Omega^{(0)} .
\end{aligned}
$$

The Dirac equation (3.11b), at the same time, is transformed into

$$
\left[\gamma_{\mu} \partial_{\mu}+m \cos 2 \alpha+i m \gamma_{5} \sin 2 \alpha\right] \psi=0 .
$$

The moral of this is that the self-consistent self-energy $\Sigma$ is determined only up to a $\gamma_{5}$ transformation. This can be easily verified from Eq. (3.4), in which the second term on the right-hand side is nonvanishing when a propagator corresponding to Eq. (3.26) is used. Although Eq. (3.26) seems to violate parity conservation, it is only superficially so since $\Omega_{\alpha}{ }^{(m)}$ is now not an eigenstate of parity. We could alternatively say that the parity operator undergoes transformation together with the mass operator. Despite the odd form of the equation (3.26), there is no change in the physical predictions of the theory. We shall see more of this later.

Let us calculate, as before, the scalar product of $\Omega_{\alpha}{ }^{(m)}$ and $\Omega_{\alpha^{\prime}}{ }^{(m)}$. From Eqs. (3.17) and (3.25) we get

$$
\begin{aligned}
& \left(\Omega_{\alpha}{ }^{(m)}, \Omega_{\alpha^{\prime}}{ }^{(m)}\right) \\
& =\prod_{p, \pm}\left[\frac{1}{2}\left(1+\beta_{p}\right)-e^{ \pm 2 i\left(\alpha^{\prime}-\alpha\right)} \frac{1}{2}\left(1-\beta_{p}\right)\right] \\
& =\prod_{p, \pm}\left[1+\left(e^{ \pm 2 i\left(\alpha^{\prime}-\alpha\right)}-1\right) \frac{1}{2}\left(1-\beta_{p}\right)\right] \\
& =\exp \left\{\sum_{p, \pm} \ln \left[1+\left(e^{ \pm 2 i\left(\alpha^{\prime}-\alpha\right)}-1\right) \frac{1}{2}\left(1-\beta_{p}\right)\right]\right\} .
\end{aligned}
$$

For large $|\mathbf{p}|$, the exponent goes like

$$
\frac{V}{(2 \pi)^{3}} \sum_{ \pm}\left(e^{ \pm 2 i\left(\alpha^{\prime}-\alpha\right)}-1\right) \int \frac{m^{2}}{4 \mathbf{p}^{2}} d^{3} p .
$$

The integral is again divergent. Hence

$$
\begin{aligned}
& \left(\Omega_{\alpha}{ }^{(m)}, \Omega_{\alpha^{\prime}}(m)\right)=\left(\Omega^{(m)}, \exp \left[-i\left(\alpha^{\prime}-\alpha\right) X\right] \Omega^{(m)}\right) \\
& =0, \quad \alpha^{\prime} \neq \alpha(\bmod 2 \pi),
\end{aligned}
$$

and, of course

$$
\left(\Omega^{(0)}, \Omega_{\alpha}^{(m)}\right)=0 .
$$

We can evaluate $\left(\Omega_{\alpha}{ }^{(m)}, \Omega_{\alpha^{\prime}}{ }^{(m)}\right)$ alternatively from Eqs. (3.22) and (3.24). Then

$$
\sum_{m=-\infty}^{\infty}\left|C_{2 n}\right|^{2} e^{2 n i\left(\alpha-\alpha^{\prime}\right)}=0, \quad \alpha \neq \alpha^{\prime}(\bmod 2 \pi)
$$

implying that

$$
\left|C_{0}\right|=\left|C_{ \pm 2}\right|=\left|C_{ \pm 4}\right|=\cdots=C .
$$

Thus there is an infinity of equivalent worlds described by $\Omega_{\alpha}{ }^{(m)}, 0 \leqslant \alpha<2 \pi$. The states $\Omega_{2 n}$ of Eq. (3.22) are then expressed in terms of $\Omega_{\alpha}{ }^{(m)}$ as

$$
C_{2 n} \Omega_{2 n}{ }^{(m)}=\frac{1}{2 \pi} \int_{0}^{2 \pi} e^{2 n i \alpha} \Omega_{\alpha}{ }^{(m)} d \alpha,
$$

which form another orthogonal set. Since the original total $H$ commutes with $X$, it will have no matrix elements connecting different "worlds." Moreover, as 
was the case with $\Omega^{(m)}$ and $\Omega^{(0)}$, no finite measurement can induce similar transitions. This is a kind of superselection rule, which effectively avoids the apparent degeneracy to show up as physical effects. ${ }^{14}$ The usual description of the world by means of $\Omega^{(m)}$ and ordinary Dirac particles must be regarded as only the most convenient one.

We still are left with some paradoxes. The $X$ conservation implies the existence of a conserved $X$ current :

$$
\begin{aligned}
j_{\mu 5} & =i \bar{\psi} \gamma_{\mu} \gamma_{5} \psi, \\
\partial_{\mu} j_{\mu 5} & =0,
\end{aligned}
$$

which can readily be verified from Eq. (2.6). On the other hand, for a massive Dirac particle the continuity equation is not satisfied:

$$
\partial_{\mu} \bar{\psi}^{(m)} \gamma_{\mu} \gamma_{5} \psi^{(m)}=2 m \bar{\psi}^{(m)} \gamma_{5} \psi^{(m)}
$$

If a massive Dirac particle has to be a real eigenstate of the system, how can this be reconciled? The answer would be that the $X$-current operator taken between real one-nucleon states should not be given simply by $i \gamma_{\mu} \gamma_{5}$ because of the "radiative corrections." We expect instead

$$
\left\langle p^{\prime}\left|j_{\mu 5}\right| p\right\rangle=\bar{u}\left(p^{\prime}\right) X_{\mu}\left(p^{\prime}, p\right) u(p),
$$

where the renormalized quantity $X_{\mu 5}$ should be, from relativistic invariance grounds, of the form

$$
\begin{gathered}
X_{\mu}\left(p^{\prime}, p\right)=F_{1}\left(q^{2}\right) i \gamma_{\mu} \gamma_{5}+F_{2}\left(q^{2}\right) \gamma_{5} q_{\mu}, \\
q=p^{\prime}-p, \quad p^{2}=p^{\prime 2}=-m^{2} .
\end{gathered}
$$

The continuity equation $\left(3.32^{\prime}\right)$, together with Eq. (3.33), further reduces this to

$$
\begin{aligned}
F_{1} & =F_{2} q^{2} / 2 m \equiv F \\
X_{\mu}\left(p^{\prime}, p\right) & =F\left(q^{2}\right)\left(i \gamma_{\mu} \gamma_{5}+\frac{2 m \gamma_{5} q_{\mu}}{q^{2}}\right) .
\end{aligned}
$$

The real nucleon is not a point particle. Its $X$-current (3.36) is provided with the dramatic "anomalous" term.

To understand the physical meaning of the anomalous term, we have to make use of the dispersion relations. The form factors $F_{1}$ and $F_{2}$ will, in general, satisfy dispersion relations of the form

$$
F_{i}\left(q^{2}\right)=F_{i}(0)-\frac{q^{2}}{\pi} \int \frac{\operatorname{Im} F_{i}\left(-\kappa^{2}\right)}{\left(q^{2}+\kappa^{2}-i \epsilon\right) \kappa^{2}} d \kappa^{2},
$$

assuming one subtraction. Each singularity at $\kappa^{2}$ corresponds to some physical intermediate state. Thus if $F(0) \neq 0$, Eq. (3.36) indicates that there is a pole at $q^{2}=0$ for $F_{2}$ (and no subtraction), which means in turn that there is an isolated intermediate state of zero mass.

${ }^{14}$ This was discussed by R. Haag, Kgl. Danske Videnskab. Selskab, Mat.-fys. Medd. 29, No. 12 (1955). See also L. van Hove, Physica 18, 145 (1952).

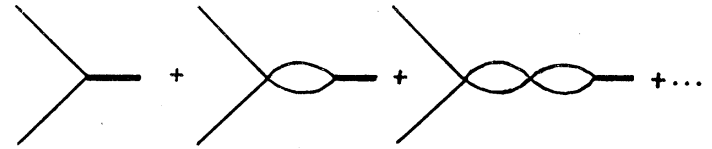

FIG. 2. Graphs corresponding to the Bethe-Salpeter equation in "ladder" approximation. The thick line is a bound state.

To see its nature, we take a time-like $q$ in its own rest frame and go to the limit $q^{2} \rightarrow 0$. The anomalous term has then only the time component, and is proportional to the amplitude for creation of a nucleon pair in a $J=0^{-}$ state. Hence the zero mass state must have the same property as this pair. It belongs to nucleon number zero, so that we may call it a zero-mass pseudoscalar meson. In order for a $\gamma_{5}$-invariant Hamiltonian such as Eq. (2.6) to allow massive nucleon states and a nonvanishing $X$ current for $q=0$, it is therefore necessary to have at the same time pseudoscalar zero-mass mesons coupled with the nucleons. Since we did not have such mesons in the theory, they must be regarded as secondary products, i.e., bound states of nucleon pairs. This conclusion would not hold if in Eq. (3.36) $F\left(q^{2}\right)=O\left(q^{2}\right)$ near $q^{2}=0$. A nucleon then would have always $X=0$. Such a possibility cannot be excluded. We will show, however, that the pseudoscalar zero-mass bound states do follow explicitly, once we assume the nontrivial solution of the self-energy equation.

\section{THE COLLECTIVE STATES}

From the general discussion of Secs. 2 and 3, we may expect the existence of collective states of the fundamental field which would manifest themselves as stable or unstable particles. In particular we have argued that, as a consequence of the $\gamma_{5}$ invariance, a pseudoscalar zero-mass state must exist. We want now to discuss the problem in detail, trying to determine the mass spectrum of the collective excitations (at least its general features) and the strength of their coupling with the nucleons. These states must be considered as a direct effect of the same primary interaction which produces the mass of the nucleon, which itself is a collective effect. We will study the bound-state problem through the use of the Bethe-Salpeter equation, taking into account explicitly the self-consistency conditions. We first verify in the following the existence of the zero-mass pseudoscalar state.

The Bethe-Salpeter equation for a bound pair $B$ deals with the amplitude

$$
\Phi(x, y)=\langle 0|T(\psi(x) \bar{\psi}(y))| B\rangle .
$$

As is well known, the equation is relatively easy to handle in the ladder approximation. In our case we have a four-spinor point interaction and the analog of the "ladder" approximation would be the iteration of the simplest closed loop (see Fig. 2) in which all lines represent dressed particles. We introduce the vertex function 
$\Gamma$ related to $\Phi$ by

$\Phi(p)=S_{F}^{(m)}\left(p+\frac{1}{2} q\right) \Gamma\left(p+\frac{1}{2} q, p-\frac{1}{2} q\right) S_{F}^{(m)}\left(p-\frac{1}{2} q\right)$.

All we have to do then is to set up the integral equation generated by the chain of diagrams, looking for solutions having the symmetry properties of a pseudoscalar state. This means that our solutions must be proportional to $\gamma_{5}$. This requirement makes only the pseudoscalar and axial vector part of the interaction contribute to the integral equation. We have

$$
\begin{aligned}
& \Gamma\left(p+\frac{1}{2} q, p-\frac{1}{2} q\right) \\
& =\frac{2 i g_{0}}{(2 \pi)^{4}} \gamma_{5} \int \operatorname{Tr}\left[\gamma_{5} S_{F}^{(m)}\left(p^{\prime}+\frac{1}{2} q\right)\right. \\
& \left.\quad \times \Gamma\left(p^{\prime}+\frac{1}{2} q, p^{\prime}-\frac{1}{2} q\right) S_{F}^{(m)}\left(p^{\prime}-\frac{1}{2} q\right)\right] d^{4} p^{\prime} \\
& \quad-\frac{i g_{0}}{(2 \pi)^{4}} \gamma_{5} \gamma_{\mu} \int \operatorname{Tr}\left[\gamma_{5} \gamma_{\mu} S^{(m)}\left(p^{\prime}+\frac{1}{2} q\right)\right. \\
& \left.\quad \times \Gamma\left(p^{\prime}+\frac{1}{2} q, p^{\prime}-\frac{1}{2} q\right) S_{F}^{(m)}\left(p^{\prime}-\frac{1}{2} q\right)\right] d^{4} p^{\prime} .
\end{aligned}
$$

For the moment let us ignore the pseudovector term on the right-hand side. It then follows that the equation has a constant solution $\Gamma=C \gamma_{5}$ if $q^{2}=0$. To see this, first observe that for the special case $q=0$, Eq. (4.3) reduces to

$$
1=-\frac{8 i g_{0}}{(2 \pi)^{4}} \int \frac{d^{4} p}{p^{2}+m^{2}-i \epsilon},
$$

which is nothing but the self-consistency condition (3.7), provided that the same cutoff is applied. Since the pseudoscalar term of Eq. (4.3) gives a function of $q^{2}$ only, the same condition remains true as long as $q^{2}=0$.

When the pseudovector term is included, we have still the same eigenvalue $q^{2}=0$ with a solution of the form $\Gamma=C \gamma_{5}+i D \gamma_{5} \gamma \cdot q$, which is not difficult to verify (see Appendix).

We now add some remarks. First, the bound state amplitude for this solution spreads in space over a region of the order of the fermion Compton wavelength $1 / m$ because of Eq. (4.2), making the zero-mass particle only partially localizable. We want also to stress the role played by the $\gamma_{5}$ invariance in the argument. We had in fact already inferred the existence of the pseudoscalar particle from relativistic and $\gamma_{5}$ invariance alone, and at first sight the same result seems to follow now essentially from the self-consistency equation. However, we must notice that only the scalar term of the Lagrangian appears in this equation while only the pseudoscalar part contributes in the Bethe-Salpeter equation. It is because of the $\gamma_{5}$-invariant Lagrangian that the BetheSalpeter equation can be reduced to the self-consistency condition.

Along the same line we could try to see whether other bound states exist in the "ladder" approximation. However, besides calculating the spectrum, it is also im- portant to determine the interaction properties of these collective states with the fermions. For this purpose the study of the two-"nucleon" scattering amplitude appears much more suitable, as we shall realize after the following remark. Once we have recognized that in the ladder approximation the collective states would appear as real stable particles, we must expect to the same degree of approximation poles in the scattering matrix of two nucleons corresponding to the possibility of the virtual exchange of these particles. For definiteness we shall refer again as an example to the pseudoscalar zeromass particle. Let us indicate by $J_{p}(q)$ the analytical expression corresponding to the graph whose iteration produces the bound state [Fig. 3(a)]. We construct next the scattering matrix generated by the exchange of all possible simple chains built with this element. This means that we consider the set of diagrams in Fig. 3(b). The series is easily evaluated and we obtain

$$
2 g_{0} i \gamma_{5} \frac{1}{1-J_{p}(q)} i \gamma_{5},
$$

where the $\gamma_{5}$ 's refer to the pairs $\left(1,1^{\prime}\right)$ and $\left(2,2^{\prime}\right)$, respectively. The meaning of this result is clear: because of the self-consistent equation $J_{p}(0)=1$, Eq. (4.5) is equivalent to a phenomenological exchange term where the intermediate particle is our pseudoscalar massless boson (Fig. 4). The coupling constant $G$ can now be evaluated by straightforward comparison. Before doing this calculation we need the explicit expression of $J_{P}(q)$. Using the ordinary rules for diagrams, we have

$$
\begin{aligned}
J_{P}(q)= & -\frac{2 i g_{0}}{(2 \pi)^{4}} \\
& \times \int \frac{4\left(m^{2}+p^{2}\right)-q^{2}}{\left[\left(p+\frac{1}{2} q\right)^{2}+m^{2}\right]\left[\left(p-\frac{1}{2} q\right)^{2}+m^{2}\right]} d^{4} p .
\end{aligned}
$$

It is however more convenient to rewrite $J_{P}$ in the form of a dispersive integral, and if we forget for a moment that it is a divergent expression, a simple manipulation gives

$$
\begin{gathered}
J_{P}(q)=\frac{g_{0}}{4 \pi^{2}} \int_{4 m^{2}}^{\Lambda^{2}} \frac{\kappa^{2}\left(1-4 m^{2} / \kappa^{2}\right)^{\frac{1}{2}}}{q^{2}+\kappa^{2}} d \kappa^{2} . \\
i \gamma_{5} \gamma_{5}
\end{gathered}
$$

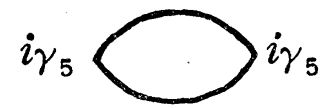

(a)

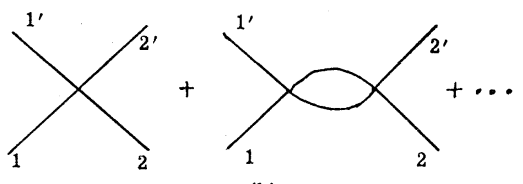

(b)

FIG. 3. The bubble graph for $J_{P}$ and the scattering matrix generated by it. 
In order for this expression to be meaningful, a new cutoff $\Lambda$ must be introduced. There is no simple relation between this and the previous cutoffs. The dispersive form is more comfortable to handle and accordingly we shall reformulate the self-consistent condition $J_{P}(0)=1$, or

$$
1=\frac{g_{0}}{4 \pi^{2}} \int_{4 m^{2}}^{\Lambda^{2}}\left(1-4 m^{2} / \kappa^{2}\right)^{\frac{1}{2}} d \kappa^{2} .
$$

It may be of interest to remark at this point that Eq. (4.7) can be obtained also if we think of our theory as a theory with intermediate pseudoscalar boson in the limit of infinite boson mass. We are now in a position to evaluate the phenomenological coupling constant $G$. From Eqs. (4.6') and (4.7) we have

$$
J_{P}\left(q^{2}\right)=1-q^{2} \frac{g_{0}}{4 \pi^{2}} \int_{4 m^{2}}^{\Lambda^{2}} \frac{\left(1-4 m^{2} / \kappa^{2}\right)^{\frac{1}{2}}}{q^{2}+\kappa^{2}} d \kappa^{2},
$$

which leads immediately to the result

$$
\frac{G_{P}^{2}}{4 \pi}=2 \pi\left[\int_{4 m^{2}}^{\Lambda^{2}} \frac{\left(1-4 m^{2} / \kappa^{2}\right)^{\frac{1}{2}}}{\kappa^{2}} d \kappa^{2}\right]^{-1} .
$$

This equation is interesting since it establishes a connection between the phenomenological constant $G_{P}$ and the cutoff independently of the value of the fundamental coupling $g_{0}$. This fact exhibits the purely dynamical origin of the phenomenological coupling $G_{P}$. Actually $g_{0}$ is buried in the value of the mass $m$.

So far we have exploited only the $\gamma_{5}$ vertex. What happens then if the scalar part is iterated to form chains of bubbles similar to those we have already discussed? The procedure just explained can be followed again, and a quantity $J_{S}(q)$ can be defined similarly with the result

$$
J_{S}(q)=\frac{g_{0}}{4 \pi^{2}} \int_{4 m^{2}}^{\Lambda^{2}} \frac{\left(\kappa^{2}-4 m^{2}\right)\left(1-4 m^{2} / \kappa^{2}\right)^{\frac{1}{2}}}{q^{2}+\kappa^{2}} d \kappa^{2} .
$$

It is immediately seen that because of Eq. (4.7)

$$
J_{S}\left(-4 m^{2}\right)=1
$$

which causes a new pole to appear in the $S$ matrix for $q^{2}=-4 m^{2}$. This means that we have another collective state of mass $2 m$, parity + and spin 0 ! We observe that it is necessary to assume the same cutoff as in the pseudoscalar case in order that this result may be obtained. The choice of the same cutoff in both cases seems to be suggested by the $\gamma_{5}$ invariance as will be seen later. We also notice the peculiar symmetry existing between the pseudoscalar and the scalar state: the first has zero mass and binding energy $2 m$, while the opposite is true for the scalar particle. So in the boundstate picture the scalar particle would not be a true bound state and should be, rather, interpreted as a
FIG. 4. The equivalent phenomenological onemeson exchange graph.

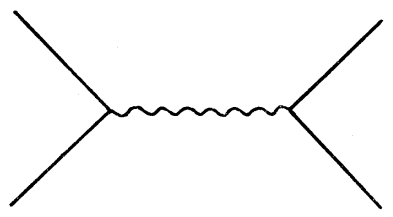

correlated exchange of pairs in the scattering process. ${ }^{15}$ The "nucleon-nucleon" forces induced by the exchange of the scalar particle are, of course, of rather short range. The general physical implications of these results will be discussed more thoroughly later.

The phenomenological coupling constant $G_{S}$ for the scalar meson is given by

$$
\frac{G_{S^{2}}}{4 \pi}=2 \pi\left[\int_{4 m^{2}}^{\Lambda^{2}} \frac{\left(1-4 m^{2} / \kappa^{2}\right)^{\frac{1}{2}}}{\left(\kappa^{2}-4 m^{2}\right)} d \kappa^{2}\right]^{-1} .
$$

Let us next turn to the vector state generated by iteration of the vector interaction. In this case we obtain for each "bubble" a tensor

$$
\begin{aligned}
& J_{V \mu \nu}=\left(\delta_{\mu \nu}-q_{\mu} q_{\nu} / q^{2}\right) J_{V} \\
& J_{V}=-\frac{g_{0}}{4 \pi^{2}} \frac{q^{2}}{3} \int_{4 m^{2}}^{\Lambda^{2}} \frac{d \kappa^{2}}{q^{2}+\kappa^{2}} \\
& \\
& \quad \times\left(1+\frac{2 m^{2}}{\kappa^{2}}\right)\left(1-4 m^{2} / \kappa^{2}\right)^{\frac{1}{2}} .
\end{aligned}
$$

Perhaps a remark is in order here regarding the evaluation of $J_{V}$. It suffers from an ambiguity of subtraction well known in connection with the photon self-energy problem. The above result is of the conventional gauge invariant form, which we take to be the proper choice.

Equation (4.13) leads to the scattering matrix

$$
g_{0}\left[\gamma_{\mu} \frac{1}{1-J_{V}} \gamma_{\mu}-\gamma \cdot q \frac{J_{V}}{\left(1-J_{V}\right) q^{2}} \gamma \cdot q\right],
$$

where the second term is, of course, effectively zero. It can be easily seen that the denominator can produce a pole below $4 m^{2}$ for sufficiently small $\Lambda^{2}$. In fact, from Eqs. (4.7) and (4.13), we find

$$
(8 / 3) m^{2}<\mu_{V}^{2}
$$

The coupling constant is given by

$$
\frac{G_{V^{2}}}{4 \pi}=3 \pi\left[\int_{4 m^{2}}^{\Lambda^{2}} d \kappa^{2} \frac{\kappa^{2}+2 m^{2}}{\left(\kappa^{2}-\mu_{V}^{2}\right)^{2}}\left(1-4 m^{2} / \kappa^{2}\right)^{\frac{1}{2}}\right]^{-1} .
$$

It must be noted that the mass of the vector meson now depends on the cutoff, unlike the previous two cases.

Finally we are left with the pseudovector state. We

${ }^{15}$ Of course this and other heavy mesons will in general become unstable in higher order approximation, which is beyond the scope of the present paper. 
find for the bubble ${ }^{16}$

$$
\begin{aligned}
J_{A \mu \nu} & =-J_{V \mu \nu}+J_{A}{ }^{\prime} \delta_{\mu \nu}, \\
J_{A}{ }^{\prime} & =\frac{g_{0} m^{2}}{2 \pi^{2}} \int_{4 m^{2}}^{\Lambda^{2}} \frac{d \kappa^{2}}{q^{2}+\kappa^{2}}\left(1-4 m^{2} / \kappa^{2}\right)^{\frac{1}{2}} .
\end{aligned}
$$

In view of the self-consistency condition (4.7), it can be seen that this does not produce a pole of the scattering matrix for $-q^{2}<4 m^{2}$, corresponding to a pseudovector meson.

So far we have considered only iterations of the same kind of interactions. In the ladder approximation there is actually a coupling between pseudoscalar and pseudovector interactions as was explicitly considered in Eq. (4.3). However, the coupling between scalar and vector interactions vanish because of the Furry's theorem.

This coupling of pseudoscalar and pseudovector interactions does not change the pion pole of the scattering matrix, but it affects the coupling of the pion to the nucleon since a chain of the pseudoscalar can join the external nucleon with an axial vector interaction. In other words, the pion-nucleon coupling is in general a mixture of pseudoscalar and derivative pseudovector types (Appendix).

We would like to inject here a remark concerning the trivial solution of the self-energy equation, against which we had no decisive argument. So let us also try to apply our scattering formula to this solution. For the pseudoscalar state we now find $J_{P}(q=0)>1$, provided that the cutoff $\Lambda$ is kept fixed and $m$ is set equal to zero in Eq. $\left(4.6^{\prime}\right)$. (The pseudovector interference vanishes.) In other words, there will be a pole for some $q^{2}>0\left(\mu^{2}<0\right)$. This is again a supporting evidence that the trivial solution could be unstable, capable of decaying by emitting such mesons. The final answer, however, depends on the exact nature of the cutoff.

Finally we would like to discuss the nucleon-nucleon scattering in the same spirit and approximation as for the nucleon-antinucleon scattering. In order to make a correspondence with the previous cases, it is convenient to rewrite the Hamiltonian in the following way:

$$
\begin{aligned}
H_{1} & =-g_{0}\left[\bar{\psi} \psi \bar{\psi}^{c} \psi^{c}-\bar{\psi} \gamma_{5} \psi \bar{\psi}^{c} \gamma_{5} \psi^{c}\right] \\
& =\frac{1}{2} g_{0}\left[\bar{\psi} \gamma_{\mu} \psi^{c} \bar{\psi}^{c} \gamma_{\mu} \psi-\bar{\psi} \gamma_{\mu} \gamma_{5} \psi^{c} \bar{\psi}^{c} \gamma_{\mu} \gamma_{5} \psi\right] \\
& =-\frac{1}{2} g_{0}\left[\bar{\psi} \gamma_{\mu} C \bar{\psi} \psi C^{-1} \gamma_{\mu} \psi-\bar{\psi} \gamma_{\mu} \gamma_{5} C \bar{\psi} \psi C^{-1} \gamma_{\mu} \gamma_{5} \psi\right]
\end{aligned}
$$

where $\psi^{c}, \bar{\psi}^{c}$ are the charge-conjugate fields.

The last form of Eq. (4.18) is suitable for our purpose. We note first that the vector part of the interaction is identically zero because of the anticommutativity of $\psi$. Thus only the pseudovector part survives. A "bubble" made of this interaction then is seen to give rise to the same integral $J_{A}$, Eq. (4.17). Since the interfering pseudoscalar interaction is missing in the present case,

${ }^{16} \mathrm{We}$ meet here again the problem of subtraction. Our choice follows naturally from comparison with the vector case, and is consistent with Eq. (3.33).
TABLE I. Mass spectrum.

\begin{tabular}{cccc}
\hline \hline $\begin{array}{c}\text { Nucleon } \\
\text { number }\end{array}$ & Mass $\mu$ & $\begin{array}{c}\text { Spin- } \\
\text { parity }\end{array}$ & $\begin{array}{c}\text { Spectroscopic } \\
\text { notation }\end{array}$ \\
\hline 0 & 0 & $0^{-}$ & ${ }^{1} S_{0}$ \\
0 & $2 m$ & $0^{+}$ & ${ }^{3} P_{0}$ \\
0 & $(8 / 3) m^{2}<\mu^{2}$ & $1^{-}$ & ${ }^{3} P_{1}$ \\
\pm 2 & $2 m^{2}<\mu^{2}$ & $0^{+}$ & ${ }^{1} S_{0}$ \\
\hline
\end{tabular}

we get the complete scattering matrix by iterating $J_{A}$ :

$$
\begin{aligned}
& -\gamma_{\mu} \gamma_{5} C\left[\frac{\delta_{\mu \nu}-q_{\mu} q_{\nu} / q^{2}}{1-J_{A}}+\frac{q_{\mu} q_{\nu} / q^{2}}{1-J_{A}{ }^{\prime}}\right] C^{-1} \gamma \gamma_{5} \\
& =\gamma_{\mu} \gamma_{5} C \frac{1}{1-J_{A}} C^{-1} \gamma_{\mu} \gamma_{5} \\
& +\gamma \cdot q \gamma_{5} C \frac{J_{V} / q^{2}}{\left(1-J_{A}{ }^{\prime}\right)\left(1-J_{A}\right)} C^{-1} \gamma \cdot q \gamma_{5} \\
& J_{A} \equiv J_{A}{ }^{\prime}-J_{V} .
\end{aligned}
$$

The first term, corresponding to a scattering in the $J=1^{-}$state, does not have a pole. The second term can have one below $4 m^{2}$ for $1=J_{A}{ }^{\prime}$. With Eqs. (4.7) and (4.17), this determines the mass $\mu_{D}$ :

$$
2 m^{2}<\mu_{D}^{2} \text {. }
$$

In this second term of the scattering matrix, the wave function is proportional to $C \gamma \cdot q \gamma_{5}$, so that the bound state behaves like a scalar "deuteron" (a singlet $S$ state). The residue of the pole determines the nucleon"deuteron" coupling constant (derivative) $G_{D}{ }^{2}$, which is positive as it should be.

Table I summarizes the main results of this section. Although our approximation is a very crude one, we believe that it reflects the real situation at least qualitatively, because all the results are understandable in simple physical terms. Thus in the nonrelativistic sense, our Hamiltonian contains spin-independent attractive scalar and vector interactions plus a spin-dependent axial vector interaction between a particle and an antiparticle. Between particles, the vector part turns into a repulsion. Table $I$ is just what we expect for the level ordering from this consideration.

\section{PHENOMENOLOGICAL THEORY AND $\gamma_{5}$ INVARIANCE}

In the previous section special subsets of diagrams were taken into account, and the existence of various boson states was established, together with their couplings with the nucleons. As was discussed there, we can reasonably expect that these results are essentially correct in spite of the very simple approximations. Because the bosons have in general small masses (compared to the unbound nucleon states), they will play important roles in the dynamics of strong interactions at least at energies comparable to these masses. 
DYNAMICAL MODEL OF

Thus if we are willing to accept the conclusions of our lowest order approximation, what we should do then is to study the dynamics of systems consisting of nucleons and the different kinds of bosons which all together represent the primary manifestation of the fundamental interaction. These particles will be now assumed to interact via their phenomenological couplings. So we may describe our purpose as an attempt to construct a theory in the conventional sense in which a separate field is introduced for each kind of particle. However, this is not a simple and unambiguous problem because our fundamental theory is completely $\gamma_{5}$ invariant and we must make sure that this invariance is preserved at any stage of our calculations in order that the results be meaningful. For a better understanding of the problem, let us consider our Lagrangian in the lowest selfconsistent approximation. We have

where

$$
L^{\prime}=L_{0}{ }^{\prime}+L_{I}^{\prime}
$$

$$
\begin{aligned}
L_{0}{ }^{\prime} & =-\left(\bar{\psi} \gamma_{\mu} \partial_{\mu} \psi+m \bar{\psi} \psi\right), \\
L_{I}{ }^{\prime} & =g_{0}\left[(\bar{\psi} \psi)^{2}-\left(\bar{\psi} \gamma_{5} \psi\right)^{2}\right]+m \bar{\psi} \psi
\end{aligned}
$$

$L^{\prime}$ is obviously $\gamma_{5}$ invariant. In order to preserve this invariance we must study the $S$ matrix generated by $L_{I}{ }^{\prime}$. Some subsets of diagrams have been considered in the previous section and it will be shown now how those calculations comply with $\gamma_{5}$ invariance. This point must be understood clearly so that we shall discuss it in a rather systematic way. Let us recall first how we constructed the scattering matrix in the "ladder" approximation. The lowest-order contribution is certainly invariant as no internal massive line appears. But what will happen to the next-order terms [Fig. 3(b)]? To these diagrams corresponds the expression

$$
J_{S}\left(q^{2}\right)-\gamma_{5} J_{P}\left(q^{2}\right) \gamma_{5}+i J_{S P}\left(q^{2}\right) \gamma_{5}+i \gamma_{5} J_{P S}\left(q^{2}\right) .
$$

In the gauge in which our calculations were performed, the last two terms happened to be zero. We write down next the transformation properties of the quantities appearing above. By straightforward calculation we find

$$
\begin{aligned}
\gamma_{5} & \rightarrow \gamma_{5} \cos 2 \alpha+i \sin 2 \alpha, \\
1 & \rightarrow \cos 2 \alpha+i \gamma_{5} \sin 2 \alpha, \\
J_{P} & \rightarrow J_{P} \cos ^{2} 2 \alpha+J_{S} \sin ^{2} 2 \alpha, \\
J_{S} & \rightarrow J_{S} \cos ^{2} 2 \alpha+J_{P} \sin ^{2} 2 \alpha, \\
J_{S P} & \rightarrow\left(J_{P}-J_{S}\right) \sin 2 \alpha \cos 2 \alpha, \\
J_{P S} & \rightarrow\left(J_{P}-J_{S}\right) \sin 2 \alpha \cos 2 \alpha .
\end{aligned}
$$

By simple substitution the invariance follows easily. The argument can now be extended to all orders, provided at each order all the possible combinations of $S$ and $P$ are included. The invariance of the scattering in the "ladder" approximation is thus established. It may look surprising that the $S P$ and $P S$ contributions do not vanish identically. This can be understood by considering the fact that the $\gamma_{5}$ transformation changes the
ELEMENTARY PARTICLES

parity of the vacuum which will be in general a superposition of states of opposite parities. In this way products of fields of different parities (as the $S P$ propagator) may have a nonvanishing average value in the vacuum state.

We may now attempt the construction of the phenomenological coupling by introducing two local fields $\Phi_{P}$ and $\Phi_{S}$ describing the pseudoscalar and the scalar particles, respectively. We start by observing that, in the same gauge in which the previous calculations were made, we can write the meson-nucleon interaction as

$$
L_{I}=G_{P} i \bar{\psi} \gamma_{5} \psi \Phi_{P}+G_{S} \bar{\psi} \psi \Phi_{S}
$$

In order to find the general expression valid in any gauge, it is convenient to introduce the following twodimensional notation

$$
\varphi \equiv\left(\begin{array}{c}
i \bar{\psi} \gamma_{5} \psi \\
\bar{\psi} \psi
\end{array}\right), \quad \Phi \equiv\left(\begin{array}{c}
\Phi_{P} \\
\Phi_{S}
\end{array}\right), \quad G \equiv\left(\begin{array}{cc}
G_{P} & 0 \\
0 & G_{S}
\end{array}\right)
$$

The interaction Lagrangian Eq. (5.4) can be written in this notation in a compact form,

$$
L_{I}=\varphi G \Phi \text {. }
$$

The effect of the $\gamma_{5}$ transformation on $\varphi$ is described with the aid of the matrix

$$
U \equiv\left(\begin{array}{cc}
\cos 2 \alpha & -\sin 2 \alpha \\
\sin 2 \alpha & \cos 2 \alpha
\end{array}\right),
$$

which satisfies $U U^{+}=U U^{-1}=U U^{T}=1$. In other words, the $\gamma_{5}$ transformation induces a unitary transformation in the two-dimensional space, and Eq. (5.6) remains invariant if

$$
G \rightarrow U G U^{-1}, \quad \Phi \rightarrow U \Phi .
$$

To complete the construction of the theory, the free Lagrangian for the fields $\Phi_{P}$ and $\Phi_{S}$ must be added. If we work again in the special gauge $\alpha=0$, we may write

$$
L_{0}=-\frac{1}{2} \partial_{\mu} \Phi_{P} \partial_{\mu} \Phi_{P}-\frac{1}{2} \partial_{\mu} \Phi_{S} \partial_{\mu} \Phi_{S}-\frac{1}{2} \mu^{2} \Phi_{S}{ }^{2},
$$

where $\mu^{2}=4 m^{2}$. We use again the two-dimensional notation, and defining the mass operator

$$
M^{2} \equiv\left(\begin{array}{cc}
0 & 0 \\
0 & \mu^{2}
\end{array}\right)
$$

we write Eq. (5.9) in the invariant form

$$
L_{0}=-\frac{1}{2} \partial_{\mu} \Phi \partial_{\mu} \Phi-\frac{1}{2} \Phi M^{2} \Phi \text {. }
$$

In this way we have given a formal prescription for the $\gamma_{5}$ transformation in the phenomenological treatment. We have to emphasize here that the Lagrangians (5.9) and (5.11) are not $\gamma_{5}$ invariant in the ordinary sense of the word. In our theory, where the mesons are only phenomenological substitutes which partially represent the dynamical contents of the theory, they may 
be, however, called $\gamma_{5}$ covariant. In other words, the masses and the coupling constants are not fixed parameters, but rather dynamical quantities which are subject to transformations when the representation is changed. It will be legitimate to ask whether this situation corresponds to the one obtained in the framework of the fundamental theory and discussed in the "ladder" approximation in the previous section. We shall examine the transformation rule for the mass operator $M^{2}$, since this illustrates the case in point. Let us calculate explicitly $M^{2}$ in an arbitrary gauge $\alpha$. We have

$$
\begin{aligned}
M^{2} \rightarrow U M^{2} U^{-1} & \\
= & \mu^{2}\left(\begin{array}{cc}
\sin ^{2} 2 \alpha & -\sin 2 \alpha \cos 2 \alpha \\
-\sin 2 \alpha \cos 2 \alpha & \cos ^{2} 2 \alpha
\end{array}\right) .
\end{aligned}
$$

The meaning of this equation is that the pseudoscalar and the scalar particle will have generally different masses in different gauges. In particular we see that the pseudoscalar particle has in the gauge $\alpha$ a mass $\sin 2 \alpha \mu$. If this is the case we must expect that after the transformation the pole in the corresponding propagator will move from $q^{2}=0$ to $q^{2}=-\left(\sin ^{2} 2 \alpha\right) \mu^{2}$. This actually may be verified directly in the "ladder" approximation which shows that the pion propagator changes according to

$$
i G_{P}^{2} \Delta_{F P}=\frac{2 g_{0}}{1-J_{P}} \rightarrow \frac{2 g_{0}}{1-J_{P} \cos ^{2} 2 \alpha-J_{S} \sin ^{2} 2 \alpha} .
$$

Using the results of the previous section, it is seen that the denominator of the right-hand side vanishes for $q^{2}=-\left(\sin ^{2} 2 \alpha\right) 4 m^{2}$. In this way we have seen how our $\gamma_{5}$-invariant theory can be approximated by a phenomenological description in terms of pseudoscalar and scalar mesons. Of course one may add the vector meson as well. Such a description does not look $\gamma_{5}$ invariant. It is only $\gamma_{5}$ covariant, and the masses and coupling constants must be understood to be matrices which, however, can be simultaneously diagonalized.

The reason for this situation is the degeneracy of the vacuum and the world built upon it. Only after combining all the equivalent but nonintersecting worlds labeled with different $\alpha$ do we recover complete $\gamma_{5}$ invariance. Nevertheless, even in a particular world we can find manifestations of the invariance, such as the zero-mass pseudoscalar meson and the conserved $\gamma_{5}$ current.

\section{THE CONSERVATION OF AXIAL VECTOR CURRENT}

In this section we will discuss another paradoxical aspect of the theory regarding the $\gamma_{5}$ invariance. In Sec. 3 we argued that the $X$ current should really be conserved, and that this is possible if a nucleon $X$ current possesses a peculiar anomalous term. We now verify the statement explicitly in our approximation.

First we have to realize that the problem is again how to keep the $\gamma_{5}$-invariant nature of the theory at every stage of approximation. It is well known in quantum electrodynamics that, in order to observe the ordinary gauge invariance, a certain set of graphs have to be combined together in a given approximation. The necessity for this is based on a general proof which makes use of the so-called Ward identity. In our present case there also exists an analog of the Ward identity. In order to derive it, let us first consider the proper selfenergy part of our fermion in the presence of an external axial vector field $B_{\mu}$ with the interaction $L_{B}=-j_{\mu 5} B_{\mu}$. The self-energy operator is now a matrix $\Sigma^{(B)}\left(p^{\prime}, p\right)$ depending on initial and final momenta. Expanding $\Sigma$ in powers of $B$, we have

$$
\Sigma^{(B)}\left(p^{\prime}, p\right)=\Sigma(p)+\Lambda_{\mu 5}\left(p^{\prime}, p\right) B_{\mu}\left(p^{\prime}-p\right)+\cdots .
$$

We readily realize that the coefficient of the second term gives the desired $X$-current vertex correction.

On the other hand, the entire Lagrangian remains invariant under a local $\gamma_{5}$ transformation if Eq. (2.3) is accompanied by

$$
B_{\mu} \rightarrow B_{\mu}-\partial_{\mu} \alpha,
$$

where $\alpha$ is now an arbitrary function. In other words,

$$
e^{i \alpha \gamma_{5} \Sigma^{(B-\partial \alpha)}} e^{i \alpha \gamma_{5}}=\Sigma^{(B)}
$$

in a symbolical way of writing. ${ }^{17}$

Expanding (6.3) after putting $B=0$, we get

$i \alpha\left(p^{\prime}-p\right)\left[\gamma_{5} \Sigma(p)+\Sigma\left(p^{\prime}\right) \gamma_{5}\right]$ $=i \alpha\left(p^{\prime}-p\right)\left(p^{\prime}-p\right)_{\mu} \Lambda_{\mu 5}\left(p^{\prime}, p\right)$,

or

$$
\gamma_{5} \Sigma(p)+\Sigma\left(p^{\prime}\right) \gamma_{5}=\left(p^{\prime}-p\right)_{\mu} \Lambda_{\mu 5}\left(p^{\prime}, p\right)
$$

The entire vertex $\Gamma_{\mu 5}=i \gamma_{\mu} \gamma_{5}+\Lambda_{\mu 5}$ then satisfies

$$
\begin{aligned}
\gamma_{5} L^{\prime}(p)+L^{\prime}\left(p^{\prime}\right) \gamma_{5} & =-\left(p^{\prime}-p\right)_{\mu} \Gamma_{\mu 5}\left(p^{\prime}, p\right), \\
L^{\prime}(p) & \equiv-i \gamma \cdot p-\Sigma(p),
\end{aligned}
$$

which is the desired generalized Ward identity. ${ }^{18}$ The right-hand side of Eq. (6.5) is the divergence of the $X$ current, while the left-hand side vanishes when $p$ and $p^{\prime}$ are on the mass shell of the actual particle. The $X$-current conservation is thus established. Moreover, the way the anomalous term arises is now clear. For if we assume $\Sigma(p)=m, \mathrm{Eq}$. (6.4) gives

$$
2 m \gamma_{5}=\left(p^{\prime}-p\right)_{\mu} \Lambda_{\mu 5}\left(p^{\prime}-p\right),
$$

so that we may write the longitudinal part of $\Lambda$ as

$$
\Lambda_{\mu 5}{ }^{(l)}\left(p^{\prime}, p\right)=2 m \gamma_{5} q_{\mu} / q^{2}, \quad q=p^{\prime}-p,
$$

which is of the desired form.

Next we have to determine what types of graphs

${ }^{17}$ We assume here that $\alpha(x)$ is different from zero only over a finite space-time region, so that the gauge of the nontrivial vacuum, which we may fix at remote past, is not affected by the transformation. The limiting process of going over to constant $\alpha$ is then ill-defined as we can see from the fact that the anomalous term in $\Gamma_{\mu 5}$ has no limit as $q \rightarrow 0$.

${ }_{18}$ See also J. Bernstein, M. Gell-Mann, and L. Michel, Nuovo cimento 16, 560 (1960). 
should be considered for $\Gamma_{\mu}$ in our particular approximation of the self-energy. Examining the way in which the relation (6.3) is maintained in a perturbation expansion, we are led to the conclusion that our self-energy represented by Fig. 5(a) gives rise to the series of vertex graphs [Fig. 5(b)]. The summation of the graphs is easily carried out to give

$$
\Lambda_{\mu 5}=i \gamma_{5} \frac{1}{1-J_{P}} J_{P A},
$$

where $J_{P}$ was obtained before [Eq. (4.8)], and

$$
\begin{aligned}
J_{P A} & =\frac{2 i g_{0}}{(2 \pi)^{4}} \int \operatorname{Tr} \gamma_{5} S(p+q / 2) \gamma_{\mu} \gamma_{5} S(p-q / 2) d^{4} p \\
& =-\frac{g_{0}}{2 \pi^{2}} i m q_{\mu} \int_{4 m^{2}}^{\Delta^{2}} \frac{d \kappa^{2}}{q^{2}+\kappa^{2}}\left(1-4 m^{2} / \kappa^{2}\right)^{\frac{1}{2}}
\end{aligned}
$$

Thus

$$
\begin{aligned}
\Gamma_{\mu 5} & =i \gamma_{\mu} \gamma_{5}+\Lambda_{\mu 5} \\
& =i \gamma_{\mu} \gamma_{5}+2 m \gamma^{5} q_{\mu} / q^{2}
\end{aligned}
$$

in agreement with the general formula. We see also that there is no form factor in this approximation.

This example will suffice to show the general procedure necessary for keeping $\gamma_{5}$ invariance. When we consider further corrections, the procedure becomes more involved, but we can always find a set of graphs which are sufficient to maintain the $X$-current conservation. We shall come across this problem in connection with the axial vector weak interactions.

\section{SUMMARY AND DISCUSSION}

We briefly summarize the results so far obtained. Our model Hamiltonian, though very simple, has been found to produce results which strongly simulate the general characteristics of real nucleons and mesons. It is quite appealing that both the nucleon mass and the pseudoscalar "pion" are of the same dynamical origin, and the reason behind this can be easily understood in terms of (1) classical concepts such as attraction or repulsion between particles, and (2) the $\gamma_{5}$ symmetry.

According to our model, the pion is not the primary agent of strong interactions, but only a secondary effect. The primary interaction is unknown. At the present stage of the model the latter is only required to have appropriate dynamical and symmetry properties, although the nonlinear four-fermion interaction, which we actually adopted, has certain practical advantages.
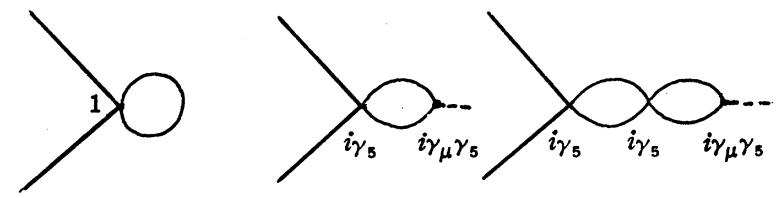

FIG. 5. Graphs for self-energy and matching radiative corrections to an axial vector vertex

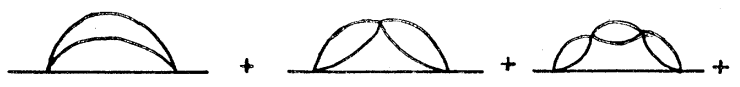

FIG. 6. A class of higher order self-energy graphs.

In our model the idealized "pion" occupies a special position in connection with the $\gamma_{5}$-gauge transformation. But there are also other massive bound states which may be called heavy mesons and deuterons. The conventional meson field theory must be regarded, from our point of view, as only a phenomenological description of events which are actually dynamic processes on a higher level of understanding, in the same sense that the phonon field is a phenomenological description of interatomic dynamics.

Our theory contains two parameters, the primary coupling constant and the cutoff, which can be translated into observed quantities: nucleon mass and the pion-nucleon coupling constant. It is interesting that the pion coupling depends only on the cutoff in our approximation. In order to make the pion coupling as big as the observed one $(\approx 15)$ the cutoff has to be rather small, being of the same order as the nucleon mass.

We would like to make some remarks about the higher order approximations. If the higher order corrections are small, the usual perturbation calculation will be sufficient. If they are large compared to the lowest order estimation, the self-consistent procedure must be set up, including these effects from the beginning. This is complicated by the fact that the pions and other mesons have to be properly taken into account.

To get an idea about the importance of the corrections, let us take the next order self-energy graph (Fig. 6). This is only the first term of a class of corrections shown in Fig. 6, the sum of which we know already to give rise to an important collective effect, i.e., the mesons. It would be proper, therefore, to consider the entire class put together. The correction is then equivalent to the ordinary second order self-energy due to mesons, plus modifications arising at high momenta. Thus strict perturbation with respect to the bare coupling $g_{0}$ will not be an adequate procedure. Evaluating, for example, the pion contribution in a phenomenological way, we get

$$
\frac{\delta m}{m}=\frac{G_{P}^{2}}{32 \pi^{2}} \int_{m^{2}}^{\Lambda^{\prime 2}} \frac{d \kappa^{2}}{\kappa^{2}}\left(1-\frac{m^{2}}{\kappa^{2}}\right),
$$

where $\Lambda^{\prime}$ is an effective cutoff. Substituting $G_{P}{ }^{2}$ from Eq. (4.9), this becomes

$$
\frac{\delta m}{m}=\frac{1}{4} \int_{4 m^{2}}^{4 \Lambda^{\prime 2}} \frac{d \kappa^{2}}{\kappa^{2}}\left(1-\frac{4 m^{2}}{\kappa^{2}}\right) / \int_{4 m^{2}}^{\Lambda^{2}} \frac{d \kappa^{2}}{\kappa^{2}}\left(1-\frac{4 m^{2}}{\kappa^{2}}\right)^{\frac{1}{2}} .
$$

As $\Lambda$ and $\Lambda^{\prime}$ should be of the same order of magnitude, the higher order corrections are in general not negligible. We may point out, on the other hand, that there is a 
tendency for partial cancellation between contributions from different mesons or nucleon pairs.

We already remarked before that the model treated here is not realistic enough to be compared with the actual nucleon problem. Our purpose was to show that a new possibility exists for field theory to be richer and more complex than has been hitherto envisaged, even though the mathematics is marred by the unresolved divergence problem.

In the subsequent paper we will attempt to generalize the model to allow for isospin and finite pion mass, and draw various consequences regarding strong as well as weak interactions.

\section{APPENDIX}

We treat here, for completeness, the problem created by the coupling of pseudoscalar and pseudovector terms encountered in the text. As we have seen, such an effect is not essential for the discussion of $\gamma_{5}$ invariance, but rather adds to complication, which however naturally appears in the ladder approximation.

First let us write down the integral equation for a vertex part $\Gamma$ :

$$
\begin{aligned}
& \Gamma\left(p+\frac{1}{2} q, p-\frac{1}{2} q\right) \\
& =\gamma\left(p+\frac{1}{2} q, p-\frac{1}{2} q\right)+\frac{2 i g_{0}}{(2 \pi)^{4}} \gamma_{5} \int \operatorname{Tr}\left[\gamma_{5} S\left(p^{\prime}+\frac{1}{2} q\right)\right. \\
& \left.\quad \times \Gamma\left(p^{\prime}+\frac{1}{2} q, p-\frac{1}{2} q\right) S_{F}\left(p-\frac{1}{2} q\right)\right] d^{4} p^{\prime} \\
& -\frac{i g_{0}}{(2 \pi)^{4}} \gamma_{5} \gamma_{\mu} \int \operatorname{Tr}\left[\gamma_{5} \gamma_{\mu} S\left(p^{\prime}+\frac{1}{2} q\right)\right. \\
& \left.\quad \times \Gamma\left(p^{\prime}+\frac{1}{2} q, p-\frac{1}{2} q\right) S_{F}\left(p-\frac{1}{2} q\right)\right] d^{4} p^{\prime} .
\end{aligned}
$$

This embraces three special cases depending on the inhomogeneous term $\gamma$ :

(a) $\gamma=0$ for the Bethe-Salpeter equation for the pseudoscalar meson;

(b) $\gamma=i \gamma_{\mu} \gamma_{5}$ for the pseudovector vertex function $\Gamma_{\mu 5}$;

(c) $\gamma=2 g_{0}\left(\gamma_{5}\right)_{f}\left(\gamma_{5}\right)_{i}-g_{0}\left(\gamma_{\mu} \gamma_{5}\right)_{f}\left(\gamma_{\mu} \gamma_{5}\right)_{i}$ for the nucleonantinucleon scattering through these interactions.

Here $i$ and $f$ refer to initial and final states, and the integral kernel of Eq. (A.1) operates on the $f$ part.

We will consider them successively.

(a) We make the ansatz $\Gamma=C \gamma_{5}+i D \gamma_{5} \gamma \cdot q$. The integrals in Eq. (A.1) then reduce to the standard forms considered in the text. Making use of Eqs. (4.9), (4.17), and $(6.9)$, we get $^{16}$

$$
\begin{aligned}
C & =C-(C+2 m D) q^{2} I, \\
D & =(C+2 m D) m I, \\
I\left(q^{2}\right) & =\frac{g_{0}}{4 \pi^{2}} \int \frac{d \kappa^{2}}{q^{2}+\kappa^{2}}\left(1-\frac{4 m^{2}}{\kappa^{2}}\right)^{\frac{1}{2}},
\end{aligned}
$$

which lead to $q^{2}=0$, and $C: D=1-2 m^{2} I(0): m I(0)$. From Eq. (4.8), we have $0<2 m^{2} I(0)<\frac{1}{2}$.

$$
\text { (b) Put } \begin{aligned}
\Gamma_{\mu 5}= & \left(i \gamma_{\mu} \gamma_{5}+2 m \gamma_{5} q_{\mu} / q^{2}\right) F_{1}\left(q^{2}\right) \\
& +\left(i \gamma_{\mu} \gamma_{5}-i \gamma \cdot q \gamma_{5} q_{\mu} / q^{2}\right) F_{2}\left(q^{2}\right) .
\end{aligned}
$$

This is seen to satisfy the integral equation if

$$
\begin{aligned}
F_{1} & =1, \\
F_{2} & =J_{A}\left(q^{2}\right) /\left[1-J_{A}\left(q^{2}\right)\right], \\
J_{A}\left(q^{2}\right) & =2 m^{2} I\left(q^{2}\right)-J_{V}\left(q^{2}\right),
\end{aligned}
$$

where $J\left(q^{2}\right)$ was defined in Eq. (4.13).

On the mass shell, $\Gamma_{\mu 5}$ reduces to

$$
\begin{gathered}
\left(i \gamma_{\mu} \gamma_{5}+2 m \gamma_{5} q_{\mu} / q^{2}\right) F\left(q^{2}\right) \\
F\left(q^{2}\right)=1+F_{2}\left(q^{2}\right)=1 /\left[1-J_{A}\left(q^{2}\right)\right] .
\end{gathered}
$$

For $q^{2}=0$, we have $J\left(q^{2}\right)=0$ so that $1<F(0)=1$ / $\left[1-2 m^{2} I(0)\right]<2$.

(c) From the structure of the inhomogeneous term, it is clear that the scattering matrix is given by

$$
M=2 g_{0}\left(\Gamma_{5}\right)_{f}\left(\gamma_{5}\right)_{i}+g_{0}\left(\Gamma_{\mu 5}\right)_{f}\left(i \gamma_{\mu} \gamma_{5}\right)_{i}
$$

where $\Gamma_{5}$ is the pseudoscalar vertex function.

Again, from Eq. (A.1), $\Gamma_{5}$ is determined as

$$
\Gamma_{5}=\gamma_{5}\left[1-2 m^{2} I\left(q^{2}\right)\right] / q^{2} I\left(q^{2}\right)-m i \gamma \cdot q \gamma_{5} / q^{2},
$$

which has an entirely different behavior from the bare $\gamma_{5}$ for small $q^{2}$. The scattering matrix is then

$$
\begin{aligned}
M= & \left(\gamma_{5}\right)_{f}\left(\gamma_{5}\right)_{i} 2 g_{0}\left[1-2 m^{2} I\left(q^{2}\right)\right] / q^{2} I\left(q^{2}\right) \\
& -\left[\left(i \gamma \cdot q \gamma_{5}\right)_{f}\left(\gamma_{5}\right)_{i}-\left(\gamma_{5}\right)_{f}\left(i \gamma \cdot q \gamma_{5}\right)_{i}\right] 2 m g_{0} / q^{2} \\
& -\left(i \gamma \cdot q \gamma_{5}\right)_{f}\left(i \gamma \cdot q \gamma_{5}\right)_{i} g_{0} J_{A}\left(q^{2}\right) / q^{2}\left[1-J_{A}\left(q^{2}\right)\right] \\
& +\left(i \gamma_{\mu} \gamma_{5}\right)_{f}\left(i \gamma_{\mu} \gamma_{5}\right)_{i} g_{0} /\left[1-J_{A}\left(q^{2}\right)\right] .
\end{aligned}
$$

The first three terms have a pole at $q^{2}=0$. The coupling constants of the pseudoscalar meson are then

pseudoscalar coupling :

$$
G_{p}^{2}=2 g_{0}\left[1-2 m^{2} I(0)\right] / I(0),
$$

pseudovector coupling :

$$
\begin{aligned}
G_{p v}{ }^{2} & =g_{0} J_{A}(0) /\left[1-J_{A}(0)\right] \\
& =g_{0} 2 m^{2} I(0) /\left[1-2 m^{2} I(0)\right] .
\end{aligned}
$$

Their relative sign is such that the equivalent pseudoscalar coupling on the mass shell is

$$
G_{p}{ }^{\prime 2}=4 m^{2} g_{0}\left\{\left[\frac{1-2 m^{2} I(0)}{2 m^{2} I(0)}\right]^{\frac{1}{2}}+\left[\frac{2 m^{2} I(0)}{1-2 m^{2} I(0)}\right]^{\frac{1}{2}}\right\}^{2} .
$$

